Lauren M. Forgrave, J. Grace van der Gugten, Quyen Nguyen and Mari L. DeMarco*

\title{
Establishing pre-analytical requirements and maximizing peptide recovery in the analytical phase for mass spectrometric quantification of amyloid- $\beta$ peptides $1-42$ and $1-40$ in CSF
}

https://doi.org/10.1515/cclm-2021-0549

Received May 7, 2021; accepted November 16, 2021;

published online December 7, 2021

\section{Abstract}

Objectives: Amyloid- $\beta(A \beta)$ peptides in cerebrospinal fluid (CSF), including $A \beta 42$ (residues 1-42) and $A \beta 40$ (residues $1-40$ ), are utilized as biomarkers in the diagnostic workup of Alzheimer's disease. Careful consideration has been given to the pre-analytical and analytical factors associated with measurement of these peptides via immunoassays; however, far less information is available for mass spectrometric methods. As such, we performed a comprehensive evaluation of pre-analytical and analytical factors specific to $A \beta$ quantification using mass spectrometry.

Methods: Using our quantitative mass spectrometry assay for $A \beta 42$ and $A \beta 40$ in CSF, we investigated the potential for interference from hemolysate, bilirubin, lipids, and anti$\mathrm{A} \beta$-antibodies. We also optimized the composition of the calibrator surrogate matrix and $A \beta$ recovery during and after solid phase extraction (SPE).

Results: There was no interreference observed with total protein up to $12 \mathrm{~g} / \mathrm{L}$, hemolysate up to $10 \%(\mathrm{v} / \mathrm{v})$, bilirubin up to $0.5 \%(\mathrm{v} / \mathrm{v})$, intralipid up to $1 \%(\mathrm{v} / \mathrm{v})$, or anti- $\mathrm{A} \beta$-antibodies at expected therapeutic concentrations. For hemolysate, bilirubin and lipids, visual CSF contamination thresholds were established. In the analytical phase, $\mathrm{A} \beta$ recovery was increased by $\sim 50 \%$ via SPE solvent modifications and by over $150 \%$ via modification of the

\footnotetext{
*Corresponding author: Mari L. DeMarco, St. Paul's Hospital, 1081 Burrard St, Vancouver V6Z 1Y6, Canada; Phone: +604 682 2344, E-mail: mdmrco@mail.ubc.ca. https://orcid.org/0000-0001-92819547

Lauren M. Forgrave and Quyen Nguyen, Department of Pathology and Laboratory Medicine, University of British Columbia, Vancouver, Canada

J. Grace van der Gugten, Department of Pathology and Laboratory Medicine, St. Paul's Hospital, Providence Health Care, Vancouver, Canada
}

SPE collection plate, which also extended analyte stability in the autosampler.

Conclusions: Attention to mass spectrometric-specific preanalytical and analytical considerations improved analytical sensitivity and reproducibility, as well as, established CSF specimen acceptance and rejection criteria for use by the clinical laboratory.

Keywords: Alzheimer's disease; amyloid- $\beta$ peptides; biomarkers; mass spectrometry; pre-analytical phase; therapeutics.

\section{Introduction}

Alzheimer's disease (AD) is characterized by aggregation of amyloid- $\beta(A \beta)$ peptides and tau proteins in the brain. Decades of research have demonstrated that the changes occurring in the brain are reflected in the cerebrospinal fluid (CSF) concentrations of specific $\mathrm{A} \beta$ and tau proteoforms $[1,2]$. As a result, the antemortem diagnosis of AD has evolved from a clinical diagnosis to a clinical-biochemical model, where biomarkers, including the quantification of CSF $A \beta$ peptides and tau proteins, have been incorporated into the research diagnostic framework for $\mathrm{AD}$ [3-5]. $\mathrm{A} \beta$ peptides are generated by proteolytic cleavage of the transmembrane amyloid precursor protein, leading to two major $A \beta$ peptide proteoforms: $A \beta 40$ and $A \beta 42$, spanning residues $1-40$ and $1-42$, respectively. The concentration of A 342 in CSF is decreased by approximately $50 \%$ in individuals with $\mathrm{AD}$ compared to healthy controls [6] and thus is used as a marker of $A \beta$ pathological changes occurring in the brain [5]. On the other hand, $A \beta 40$ concentration in CSF is not correlated with disease [7]; it is used instead in a ratio with the $A \beta 42$ concentration, possibly controlling for basal production of $A \beta$ peptides and compensating for imprecision from the pre-analytical phase.

Measurement of $A \beta$ peptides in biofluids is notoriously challenging from an accuracy and reproducibility standpoint given the physiochemical properties of $\mathrm{A} \beta$, namely its 
hydrophobicity and amyloidogenicity [8]. A $\beta$ peptides are prone to adhering to surfaces, like test tubes and pipet tips [9], and have a propensity to spontaneously polymerize [10]. Thus, special care to prevent adsorption and aggregation of $A \beta$ peptides must be taken during the preanalytical phase [9, 11-13], and, as we highlight herein, the analytical phase of biomarker testing. The pre-analytical phase of biomarker testing includes controllable variables like individual preparation for testing (e.g., fasting) and specimen collection protocols (e.g., tube type), as well as uncontrollable variables like biological differences and environmental factors. The analytical phase of biomarker testing includes specimen processing (e.g., centrifugation, solid phase extraction [SPE]), and analysis (e.g., immunometric or mass spectrometric detection) [14].

For the quantification of $\mathrm{A} \beta$ peptides in CSF, preanalytical variables have been largely studied in the context of sandwich immunoassay methods [7, 11, 12, 15, 16]; however, the implementation of mass spectrometric assays, for both research and clinical care, brings with it different potential sources of error. Examples specific to $A \beta$ mass spectrometric assays include steps such as SPE recovery/ efficiency, propensity to aggregate in the SPE extraction solvent, adsorption of the analyte to the SPE collection plate and stability in the autosampler after extraction but prior to analysis. For immunoassays, pre-analytical and analytical considerations that have been shown to have a large impact on the accuracy of $A \beta$ quantification include plastics used in the pre-analytical phase, additives (e.g., detergents), centrifugation, storage temperature prior to processing, sample volume during storage and over three freeze/thaw cycles $[17,18]$. For mass spectrometry, one study noted that A 342 concentrations were reproducible (i.e., within $15 \%$ ) for CSF that had undergone up to four freeze-thaw cycles and for extracted samples stored in the autosampler at $7{ }^{\circ} \mathrm{C}$ for up to $36 \mathrm{~h}$ [19-21]. Also of consideration, and not yet characterized for mass spectrometric methods for $\mathrm{A} \beta 42$ and $\mathrm{A} \beta 40$, are the solvents and plastics used in the analytical phase (e.g., SPE elution conditions and collection plate), temperature during sample processing, optimal protein content in the surrogate matrix, and relevant endogenous and exogenous interferents (e.g., antibody therapeutics, hemolysate).

Interferents commonly studied during analytical method validation include those from the sample matrix, therapeutic molecules, metabolites introduced by pathology, and changes induced by sample storage [22]; those generally evaluated for analytes in CSF include hemolysate (from a traumatic lumbar puncture or leakage of the blood brain barrier), bilirubin, and exogenous molecules (e.g., therapeutics, supplements). For mass spectrometry, concerns related to the presence of hemolysate include the potential for falsely elevated or decreased reported analyte concentrations due to higher/lower concentration of the analyte in blood, alteration of the matrix (e.g., by higher concentrations of blood proteins and lipids) with the latter potentially leading to ion suppression/enhancement in the mass spectrometer, or altered macromolecular interaction in vitro. Also, the increased protein concentration beyond the typical physiological range for CSF can lead to unanticipated errors during the analytical phase including incorrect reagent-to-analyte ratios, incorrect $\mathrm{pH}$ after solvent additions, and saturation of chromatography media. Although less commonly encountered than CSF with hemolysate, CSF xanthochromia and hyperlipidemia also require consideration [23-25]. In the context of $\mathrm{AD}$, relevant exogenous interferents include therapeutic molecules such as anti-A $\beta$ monoclonal antibodies [26]. Anti$A \beta$-antibodies are known to interfere with quantification in immunoassays [27], however, in the context of mass spectrometry, such studies have not been reported in the literature.

Herein we evaluated the effect of hemolysate, bilirubin, lipids, total protein concentration, and anti- $A \beta$-monoclonal antibodies on the accuracy of $A \beta 42$ and $A \beta 40$ quantification in CSF measured using our previously established high performance liquid chromatography tandem mass spectrometry (HPLC-MS/MS) method [28]. Via these analyses, we established visual CSF hemolysate, icterus, and lipemia acceptance and rejection criteria for specimens submitted to our clinical laboratory for measurement of $A \beta 42$ and A $\beta 40$. Evaluation of the CSF total protein concentration was also used to optimize the composition of the surrogate matrix used for the assay calibrators. For the analytical phase, we optimized the SPE solvent additions and plastics used to (i) maximize peptide recovery, (ii) guard against spontaneous aggregation/precipitation, and (iii) extend analyte stability in the HPLC-MS/MS autosampler. Herein we investigate both the pre-analytical and analytical considerations for $A \beta$ quantification; the outcomes provide recommendations for sensitive and reproducible $A \beta$ quantification in clinical practice.

\section{Materials and methods}

\section{Material sources and equipment}

This study was undertaken with research ethics approval from Providence Health Care Research Institute (H18-03050) and complied with all relevant national regulations, institutional policies and is in accordance with the tenets of the Helsinki Declaration and its later 
amendments. CSF specimens were from lumbar punctures performed at St. Paul's Hospital in Vancouver, Canada, as part of routine care. The materials and methodology for the HPLC-MS/MS method have been previously described in detail [28]. Briefly, the workflow is as follows: $200 \mu \mathrm{L}$ of CSF was treated with guanidine $\mathrm{HCl}$ containing the internal standard (IS) solution (composed of ${ }^{15} \mathrm{~N}-\mathrm{A} \beta 40$ and ${ }^{15} \mathrm{~N}-\mathrm{A} \beta 42$ ), followed by SPE using a mixed-mode, strong cation-exchange, reversed-phase (Waters Oasis MCX $\mu$ Elution) plate, with the eluate analyzed by HPLC (Shimadzu HPLC, flow rate $0.35 \mathrm{~mL} / \mathrm{min}$, column oven at $50{ }^{\circ} \mathrm{C}$, using a $\mathrm{C} 18 \mathrm{EVO}$ analytical column [00D-4725-Y0; Phenomenex]) coupled to a triple quadrupole mass spectrometer (SCIEX 5500 QTRAP) and analyzed using Analyst software (SCIEX v.1.6). HPLC conditions were as follows: mobile phase A was $0.3 \%$ $\mathrm{NH}_{4} \mathrm{OH}$ in water and mobile phase $\mathrm{B}$ was 25:75 IPA:ACN. The gradient was: $1.0 \mathrm{~min}=15 \% \mathrm{~B} ; 5.0 \mathrm{~min}=55 \% \mathrm{~B} ; 5.5 \mathrm{~min}=95 \% \mathrm{~B} ; 8.9 \mathrm{~min}=95 \%$ $B$; $9.0 \mathrm{~min}=15 \% \mathrm{~B}$, with a total run time of $11.5 \mathrm{~min}$ [28]. Notably, the method is calibrated to the International Federation of Clinical Chemistry reference materials [28]. Additional materials and methods used within this study are described below.

\section{Surrogate matrix}

A spike and recovery experiment was used to assess the effect of protein content in the surrogate matrix (i.e., bovine serum albumin (BSA) in phosphate-buffered saline (PBS) [28]) on the recovery and quantification of $A \beta$ peptides. Surrogate matrix solutions with varying concentrations of BSA $(0-20 \mathrm{~g} / \mathrm{L})$ were spiked with synthetic A 42 and $\mathrm{A} \beta 40$ peptides at 100 and $1,000 \mathrm{ng} / \mathrm{L}$, respectively, and analyzed in triplicate.

\section{SPE conditions and plastics}

Various SPE conditions were tested with the goals of maximizing A $\beta$ recovery and reducing spontaneous aggregation/precipitation. CSF was pooled and aliquoted for SPE testing. For all SPE methods tested the same conditioning and sample loading conditions were used, while the wash and elution buffers and volumes were varied (Table 1). Conditions assayed were informed by two previous publications $[20,21]$. The eluate from the SPE was collected into a Nunc BSA-treated 96-well plate and placed in the autosampler for HPLC-MS/MS analysis. Recovery was compared as a relative percentage between the methods.

Loss of $A \beta$ peptides are known to occur in the collection and aliquot tubes, but analyte loss can also occur in the analytical phase as the sample comes in contact with surfaces like the SPE or eluate collection plates. While the options for SPE plates are limited, we examined changes in $\mathrm{A} \beta$ recovery that occurred when modifying the collection plate. We used different collection plates and/or blocked the plate by pre-treating with a $100 \mathrm{~g} / \mathrm{L}$ BSA solution for $24 \mathrm{~h}$, followed by washing three times with PBS prior to use. We tested the following polypropylene plates and pre-treatment strategy: Nunc plates (with and without pre-treatment) [260251; Thermo Fisher, Ottawa, Canada] and AntiBIND plates [02911700; Fisher Scientific, Ottawa, Canada] without pre-treatment. To mimic the SPE eluate, we added to the wells a $30 \% \mathrm{ACN}$ and $1 \% \mathrm{NH}_{4} \mathrm{OH}$ solution spiked with $\mathrm{A} \beta 42(500 \mathrm{ng} / \mathrm{L})$ and $\mathrm{A} \beta 40(5,000 \mathrm{ng} / \mathrm{L})$ and compared peak areas. $A \beta$ stability post-SPE elution in the autosampler was investigated. Eluate solutions (as described above) in the Nunc plates
Table 1: SPE workflows tested on the MCX micro-elution plate including methods informed by the literature (Method $A$ and Method $B$ with modifications), and the resultant optimized method (Method C).

\begin{tabular}{llll}
\hline & Method A [20] & Method B $^{\text {a }}$ & Method C \\
\hline Condition 1 & \multicolumn{3}{c}{$200 \mu \mathrm{L} \mathrm{MeOH}$} \\
Condition 2 & \multicolumn{3}{c}{$200 \mu \mathrm{L} 4 \% \mathrm{H}_{3} \mathrm{PO}_{4}$} \\
Load & \multicolumn{3}{c}{$600 \mu \mathrm{L}$ pre-treated sample } \\
Wash 1 & $200 \mu \mathrm{L} 4 \% \mathrm{H}_{3} \mathrm{PO}_{4}$ \\
Wash 2 & $200 \mu \mathrm{L} \mathrm{10 \%} \mathrm{ACN}$ & $200 \mu \mathrm{L}$ of $1 \%$ & $200 \mu \mathrm{L} 4 \% \mathrm{H}_{3} \mathrm{PO}_{4}$ \\
& & $\mathrm{H}_{3} \mathrm{PO}$ & \\
Elute & $2 \times 25 \mu \mathrm{L}$ & $2 \times 50 \mu \mathrm{L}$ & $2 \times 25 \mu \mathrm{L}$ \\
& $75: 15: 10$ & $65: 32.5: 2.5$ & $75: 15: 10$ \\
& $\mathrm{ACN}: \mathrm{H}_{2} \mathrm{O}: \mathrm{NH}_{4} \mathrm{OH}$ & $\mathrm{ACN}: \mathrm{H}_{2} \mathrm{O}: \mathrm{NH}_{4} \mathrm{OH}$ & $\mathrm{ACN}: \mathrm{H}_{2} \mathrm{O}: \mathrm{NH}_{4} \mathrm{OH}$ \\
\hline
\end{tabular}

${ }^{\mathrm{a}}$ Method B was informed by Leinenbach et al. [21], which had a different pre-treated sample loading volume $(180 \mu \mathrm{L})$, elution buffer constitution (75:22.5:2.5 ACN: $\left.\mathrm{H}_{2} \mathrm{O}: \mathrm{NH}_{4} \mathrm{OH}\right)$, and included drying down the sample post-elution and reconstituting before HPLC-MS/MS analysis, whereas the workflows we trialed avoided the additional complications of dry-down/reconstitution steps prior to analysis.

(with and without pre-treatment) and the AntiBIND plates were left in the autosampler at $4^{\circ} \mathrm{C}$ for $0,1.8,3.6,5.4$, and $7.2 \mathrm{~h}$ prior to analysis.

\section{Interference studies: total protein, hemolysate, bilirubin, lipids, and exogenous antibodies}

Spike and recovery experiments were performed to assess potential inference by elevated total protein concentration, hemolysate, unconjugated bilirubin, and intralipid. Visual color and appearance thresholds were set based on the sample with the highest concentration of the interferent tested that were within $\pm 20 \%$ of the $A \beta$ concentration prior to spiking the potential interferent. All analyses were performed in triplicate and using CSF pools. Percent bias was calculated relative to the control containing a blank spike, except for the total protein and endogenous hemolysate experiment (described below).

For the total protein assessment, two CSF pools were created: a 'high' pool from CSF with endogenously high total protein further spiked with human ethylenediaminetetraacetic acid (EDTA) plasma to a final total protein concentration of $12.6 \mathrm{~g} / \mathrm{L}$ and a 'low' pool from CSF with an undetectable total protein concentration (i.e., below the lower limit of the analytical measuring range of $0.01 \mathrm{~g} / \mathrm{L}$ of the immunoturbidimetric assay used (Siemens ADVIA 1800)). For each pool, the endogenous $A \beta 42$ and $A \beta 40$ concentration was determined and synthetic $A \beta 42$ and $A \beta 40$ were spiked to a final concentration of 3,000 and $20,000 \mathrm{ng} / \mathrm{L}$, respectively.

For hemolysate assessment, two different experiments were performed: one by spiking whole blood hemolysate into a CSF pool and measuring the endogenous $A \beta 42$ and $A \beta 40$ concentration, and another where individual CSF samples with a range of visible hemolysate were spiked with recombinant $A \beta 42$ and $A \beta 40$. For the former, $a$ CSF pool was spiked with hemolysate obtained from mechanical lysis of a whole blood EDTA sample at the following percent relative to CSF: $0,1,5,10,20,30,40$ and 50\% v/v. For the latter, four CSF 
specimens with various levels of visible physiological (not spiked) hemolysis contamination were selected and compared to a sample with no visual hemolysis. The $A \beta 42$ and $A \beta 40$ concentrations were analyzed after spiking with 1,000 ng/L of A 442 and 15,000 ng/L of $\mathrm{A} \beta 40$. Percent recovery of the spiked concentration of synthetic peptides was calculated relative to the sample with no visible hemolysate contamination. For assessment of icterus, bilirubin was spiked into a CSF pool in concentrations ranging from 0 to $0.5 \% \mathrm{v} / \mathrm{v}$. For assessment of lipemia, intralipid was spiked into a CSF pool with concentrations ranging from 0 to $1 \% \mathrm{v} / \mathrm{v}$. Percent bias was calculated based on $\mathrm{A} \beta$ concentration relative to the control sample.

For assessment of exogenous antibodies, a CSF pool was spiked with either an anti-A $\beta$-antibody (clone 4g8) [800704, BioLegend, San Diego, USA] or intravenous immunoglobulin (IVIg) [Gammagard Liquid, Lexington, USA] to a final concentration ranging from 50,000 to $600,000 \mathrm{ng} / \mathrm{L}$, based on a realistic physiological concentrations of therapeutic antibodies in CSF [29].

\section{Data analysis}

For pre-analytical interference studies, accuracy was assessed via the analyte concentration (signal normalized by the IS), and analytical sensitivity was additionally assessed by examining trends in the raw signal (peak area). For analyte recovery in the analytical phase, $A \beta$ recovery was assessed based on relative signal (peak area). For matrix interreference studies, $A \beta$ concentration within $\pm 20 \%$ of the expected concentration was deemed to have no bias, based on the previously established method precision [28]. The Mann-Whitney test was used to compare peak areas, with a p-value less than 0.05 considered statistically significant.

\section{Results}

\section{Surrogate matrix}

The concentration of BSA in the surrogate matrix yielding the highest recovery of $A \beta 42$ and $A \beta 40$ peptides was found to be between 1 and $2 \mathrm{~g} / \mathrm{L}$ (Figure 1), with no significant differences between these concentrations $(\mathrm{p}=0.85)$.

\section{SPE $A \beta$ recovery}

We tested three different SPE processes (Table 1). As a method from the literature frequently resulted in precipitating eluate solutions [21], we had modified the elution buffer ratios from $75 \%$ ACN to $65 \%$ (Method B in Table 1). In the development of Method C, we adjusted the second wash to include $4 \% \mathrm{H}_{3} \mathrm{PO}_{4}$. This resulted in a relative increase in peak area of $43 \%$ for $\mathrm{A} \beta 42$ and $54 \%$ for $\mathrm{A} \beta 40$ compared to Method $\mathrm{A}$, and $77 \%$ for $A \beta 42$ and $81 \%$ for $A \beta 40$ compared to Method B (Figure 2).

\section{Post-SPE A $\beta$ absorption and stability}

BSA pre-treatment of the Nunc 96-well plates had no effect on $\mathrm{A} \beta$ absorption for samples processed immediately; however, after $7 \mathrm{~h}$, pre-treatment improved recovery by 14 and $22 \%$ for $A \beta 42$ and $A \beta 40$, respectively, compared to the non-treated Nunc plate (Figure 3). Testing of the nontreated AntiBIND plates compared to the Nunc plates (both non-treated and pretreated), revealed a $150 \%$ increase in $A \beta 42$ and $A \beta 40$ recovery for samples analyzed immediately (Figure 3). From 0 to $7 \mathrm{~h}$, there was a relative decrease in

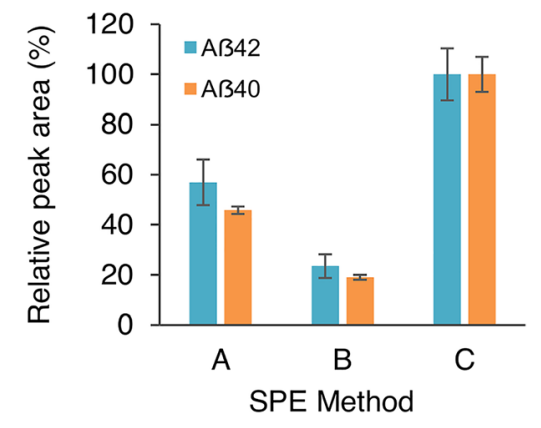

Figure 2: Optimization of the SPE conditions.

Average peak area of $A \beta 42$ and $A \beta 40$ relative to SPE method $C$, displayed as mean (SD) of three technical replicates.
A)

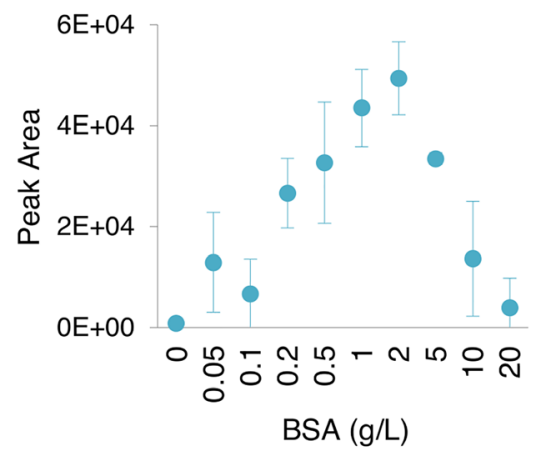

B)

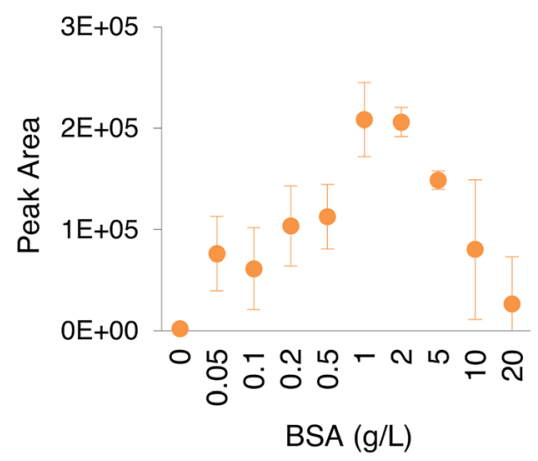

Figure 1: Optimization of the surrogate CSF matrix composition for the external calibrators. Average peak area of (A) A 42 and (B) A 440 for the surrogate matrix with increasing BSA concentrations from 0-20 g/L, displayed as mean (SD) of three technical replicates. 
A)

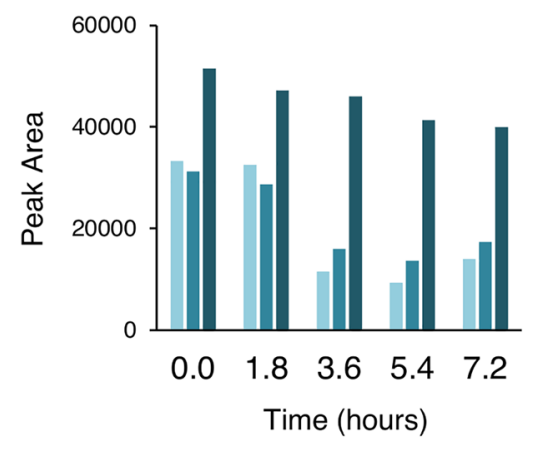

$\square$ Nunc $\approx$ Nunc pre-treated $\approx$ AntiBIND

A)

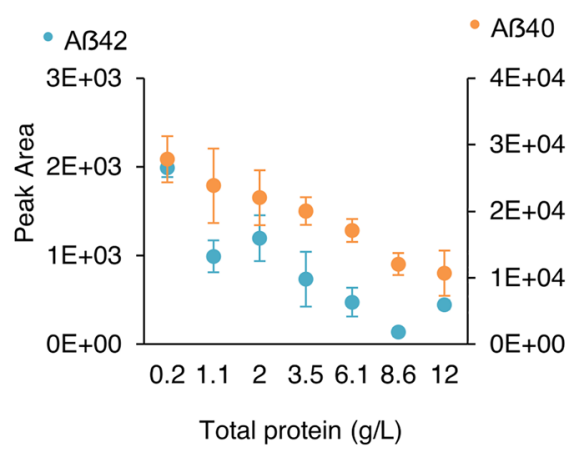

B)

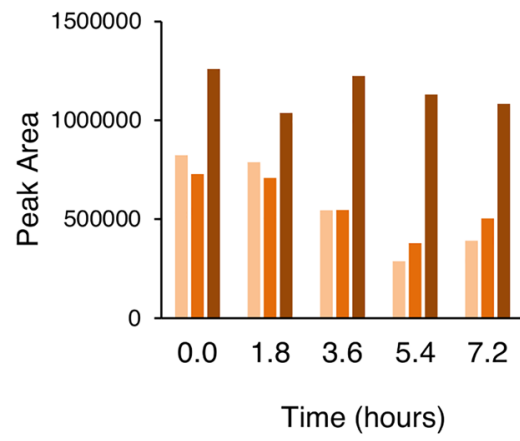

Nunc $\square$ Nunc pre-treated $\square$ AntiBIND

Figure 3: Analyte adsorption to plastics used in the analytical phase.

Peak area of (A) $A \beta 42$ and (B) $A \beta 40$ using the Nunc (with and without pre-treatment) and AntiBIND 96-well plates after storage in the autosampler prior to analysis.

Figure 4: Total protein interference experiments.

(A) Average peak area of $A \beta 42$ and A 40 in CSF with increasing total protein concentration, displayed as the mean (SD) of three technical replicates and (B) percent recovery.

peak area for $A \beta 42$ and $A \beta 40$, respectively, for all plates tested: non-treated Nunc: 58.2 and $52.5 \%$; pretreated Nunc: 44.6 and 30.8\%; and AntiBIND: 22.5 and 14.0\% (Figure 3).

\section{Interference studies}

For assessment of the effect of total protein concentration on the recovery of $A \beta$ peptides, the peak areas for both $A \beta 42$ and $A \beta 40$ showed a decreasing trend with increasing total protein concentration over the range of $0.2-12 \mathrm{~g} / \mathrm{L}$ (Figure 4A). Quantification of $A \beta 42$ and $A \beta 40$ using internal standards, however, was not affected by total protein concentration (Figure 4B).

Increasing amounts of hemolysate demonstrated a decreasing trend in $\mathrm{A} \beta$ peak areas up to $30 \%$ spiked hemolysate in the concentrations tested and was more pronounced for $\mathrm{A} \beta 42$ compared to $\mathrm{A} \beta 40$, with a decrease in peak area of 83 and 42\%, respectively (Figure 5A). There was, however, no effect on the accuracy of quantitation for up to $10 \%$ hemolysate, corresponding to a dark pink CSF sample unspun, or light pink after centrifugation (Figure 5B). The visual color and appearance of the CSF sample with $10 \%$ hemolysate was set as the preliminary threshold for sample acceptance and further confirmed by testing samples with endogenous hemolysate. Recovery of spiked concentrations of $A \beta 42$ and $A \beta 40$ into a series of samples with varying degrees of endogenous hemolysate compared to a sample with no visual hemolysate contamination demonstrated acceptable accuracy (Figure 5C).

In the matrix interference experiments for bilirubin, intralipid and immunoglobulin, there were subtle effects on raw signal intensity but no effects observed on quantification. With increasing bilirubin and intralipid concentrations the peak area for $\mathrm{A} \beta 42$ and $\mathrm{A} \beta 40$ showed an increasing trend, with no significant effect on recovery at all concentrations tested (Figure 6). Similarly, recovery was not affected in the presence of IVIg or the anti-A $\beta$-antibody, and there were no discernable trends in peak area changes by antibody concentration (Figure 7). In summary, there was no detectable interference in $A \beta 42$ and $A \beta 40$ peptide quantification at the concentrations of bilirubin, intralipid and antibodies tested. 
A)

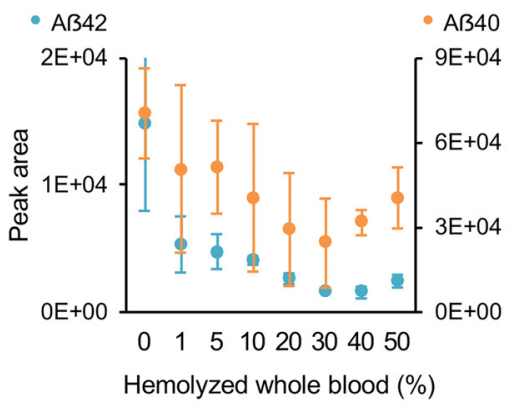

B)

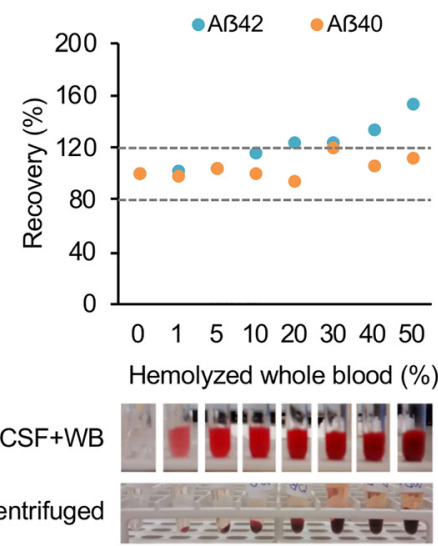

C)

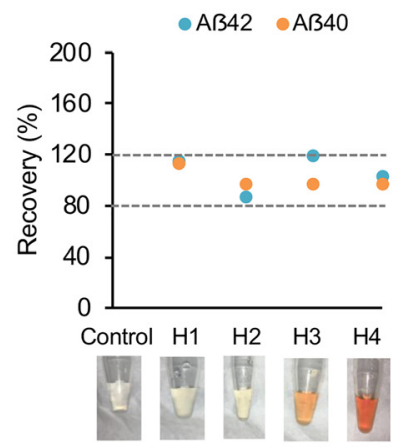

Figure 5: Hemolysate interference experiments.

(A) Average peak area for $A \beta 42$ and A 40 in pooled CSF spiked with hemolysate, data displayed as mean (SD) of three technical replicates, and (B) percent recovery. (C) Recovery of $A \beta 42$ and $A \beta 40$ spiked into CSF samples with endogenous visible hemolysate contamination.

A)

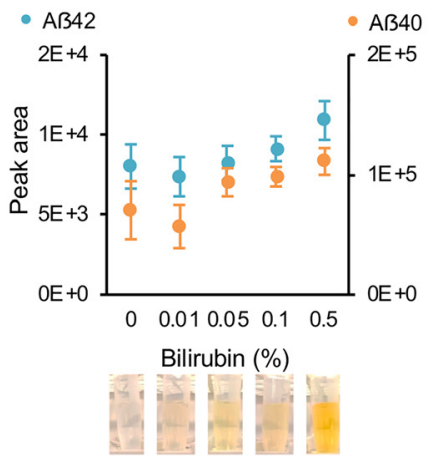

B)

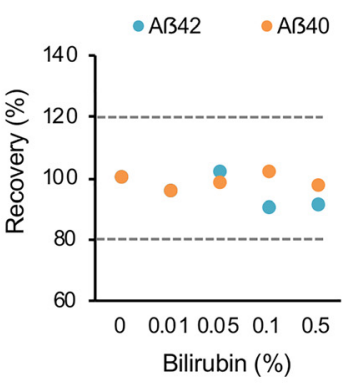

C)
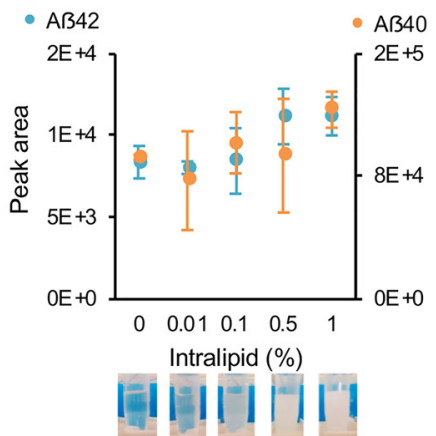

D)

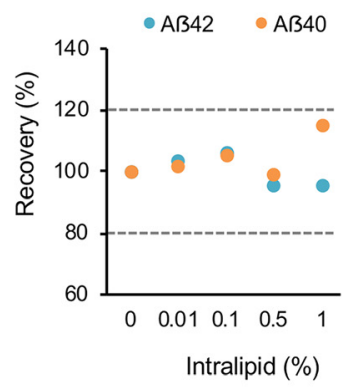

Figure 6: Bilirubin and intralipid interference experiments.

$(A \& C)$ Average peak area and (B \& D) percent recovery for $A \beta 42$ and $A \beta 40$ in pooled CSF spiked with bilirubin (A \& B) or intralipid (C \& D). Peak area displayed as the mean (SD) of three technical replicates.

A)

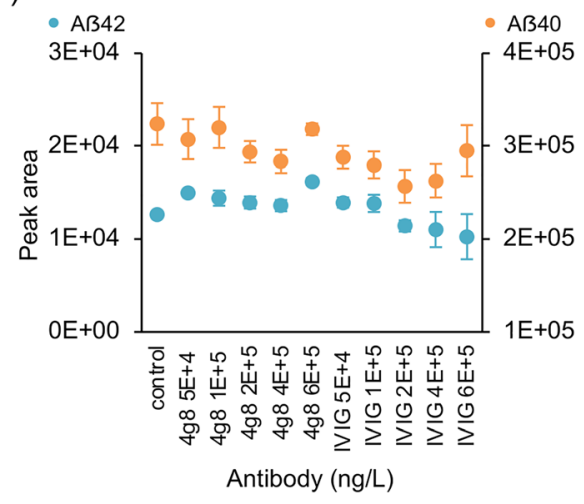

B)

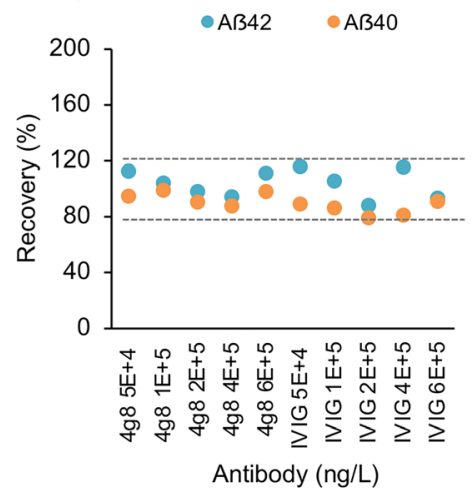

Figure 7: Exogenous antibody interference experiments.

(A) Peak area of $A \beta 42$ and $A \beta 40$ in pooled CSF with the addition of monoclonal antibody $4 \mathrm{~g} 8$ or IVIg with data displayed as mean (SD) of three technical replicates. (B) Recovery of $A \beta 42$ and $A \beta 40$. 


\section{Discussion}

Pre-analytical factors for the measurement of $\mathrm{A} \beta 42$ peptides in CSF have been extensively studied in the context of immunometric measurement. However, far less is known about pre-analytic and analytical consideration relevant to mass spectrometric methods, such as efficiency and analyte stability at specific stages of the analytical process. In this study we focused on mass spectrometry-specific preanalytical and analytical considerations for quantification of both $A \beta 42$ and $A \beta 40$ peptides using our previously described HPLC-MS/MS method [28]. This included composition of the surrogate calibrator matrix, SPE efficiency, adsorption to plastics used in the analytical phase and sample stability in the autosampler, as well as characterization of potential matrix interferents including development of visual color/clarity thresholds for CSF as this is common practice for clinical testing of CSF analytes.

In an ideal scenario, external calibrators would be generated by adding a known concentration of the analyte to the same matrix as the test samples, however, when the analyte of interest is an endogenous molecule and cannot be easily depleted, a surrogate matrix is required. Such is the case for measurement of endogenous $A \beta$ peptides in CSF by mass spectrometry. While common recipes for artificial CSF may seem like a good surrogate, they generally contain only water and buffering ions. Such solutions are not characteristic of lipid and protein-containing human CSF, and have shown to, at least for small molecule drugs, result in decreased analytical sensitivity relative to human CSF or diluted plasma [30]. For quantification of $A \beta 42$ and $A \beta 40$, a comparison between artificial CSF with and without supplementation with $5 \% \mathrm{v} / \mathrm{v}$ rat plasma revealed improved sensitivity when using the supplemented solution [20], and a subsequent study found supplementation of artificial CSF with $4 \mathrm{~g} / \mathrm{L}$ BSA was preferred over the addition of $5 \% \mathrm{v} / \mathrm{v}$ rat plasma, based on analyte recovery [19]. Herein we examined artificial CSF supplemented with a range of BSA concentrations to assess optimal surrogate matrix composition for quantification of both $A \beta 42$ and $A \beta 40$; where we observed optimal recovery of $A \beta 42$ and $A \beta 40$ in the range of $1-2 \mathrm{~g} / \mathrm{L}$ of BSA. Given the range of $0.2-0.8 \mathrm{~g} / \mathrm{L}$ of total protein concentration for CSF samples collected in a tertiary care setting excluding individuals with conditions known to alter the CSF protein concentration [31], we set the surrogate matrix for our calibrators to be $1 \mathrm{~g} / \mathrm{L}$ of BSA in $1 \times$ PBS.

Absorption to plastics is a well appreciated source of inaccuracy in $A \beta$ measurements, however, it has only been considered at the pre-analytical stage, including the type of plastic tubes used to collect or store CSF prior to analysis [17]. As an example of analytical phase considerations, SPE extraction is a common analytical step in $A \beta$ mass spectrometric methods $[20,21,28]$, but one not used in ligand binding methods. For the SPE step we considered solvent composition, quantity, and repetitions, as well as the plastic composition and conditioning of the 96-well plate used to collect the SPE eluate for mass spectrometric analysis. We found use of a wash solution containing the highest concentration of phosphoric acid tested (4\%) in combination with two small volume $(25 \mu \mathrm{L})$ elutions with 75:15:10 ACN:$\mathrm{H}_{2} \mathrm{O}: \mathrm{NH}_{4} \mathrm{OH}$, substantially increased recovery of both $\mathrm{A} \beta 42$ and $A \beta 40$ peptides over the other combinations tested. Such recovery is anticipated to contribute to increasing analytical sensitivity and precision at low analyte concentrations. We also studied the SPE collection plate itself, trialing different polypropylene plastics and blocking conditions. We found that blocking a typical 96-well plate with BSA prior to use, increased analyte recovery after prolonged storage. Also using a special low-protein binding plate (AntiBIND), increased analyte recovery without having to incorporate a blocking step, both in terms of immediate analysis of the eluate and after prolonged storage in the autosampler. Such strategies, blocking or using a specialized collection plate, as with the SPE condition optimization above, are helpful in increasing analytical sensitivity. They were also found to extend analyte stability in the autosampler, likely due to decreased rate of absorption to the plastic wells. Should there be an unanticipated delay in sample analysis post-extraction, or a run requires reinjection, the ability to analyze samples after prolonged storage in the autosampler avoids having to re-extract the entire batch.

As part of the more routine method validation experiments, we evaluated several potential sources of interferences including CSF total protein, hemolysate, lipid, bilirubin, and anti-A $\beta$-antibodies. Samples with total protein concentrations within and above the normal physiological range were tested. We found peak area decreased with increasing total protein concentration for both $A \beta 42$ and $A \beta 40$, possibly due to saturation of the SPE media; however, accuracy was not affected. For hemolysis, we tested contamination commonly seen in the clinical lab (i.e., slightly pink CSF) to concentrations far in excess of what is commonly seen. When considering hemolysate contamination of CSF in the context of immunoassays, generally the primary concern is that the increased concentration of hemoglobin will result in spectral interference; however, for mass spectrometry, hemoglobin itself is rarely the concern, but instead the gross shift in matrix 
composition in the presence of hemolysate (i.e., higher total protein and lipid concentration). As such, we added hemolysate and not hemoglobin in the first series of experiments, whereby up to $10 \% \mathrm{v} / \mathrm{v}$ whole blood hemolysate in CSF demonstrated no interference with $A \beta 42$ or $A \beta 40$ quantification. As the concentration of $A \beta 42$ and $A \beta 40$ in blood is far lower than that in CSF, the increased signal observed with increasing blood contamination is attributed to positive interference and not detection of $A \beta$ peptides from peripheral sources. As further confirmation of this finding, $A \beta$ spike and recovery experiments using individual CSF samples that contained a range of visible hemolysate after routine collection demonstrated no interference, even when hemolysate visually exceeded the $10 \%$ threshold from the hemolysate-spiking experiment. The color and appearance of the sample with $10 \%$ whole blood hemolysate was set as a visual cut-off used by our technologists. To round out common matrix interferents we examined bilirubin and lipids, finding no interference at physiological and supra-physiological concentrations.

In addition to potential endogenous interferents, plausible exogenous interferents were evaluated. AD immunotherapies, specifically anti-A $\beta$-antibodies, have been postulated to interfere with A 422 ligand binding methods [27]; however, their impact on mass spectrometric measurement has not been reported. As a surrogate for an anti$A \beta$-antibody therapeutic, we used a commercially available anti- $\mathrm{A} \beta$ monoclonal antibody whose epitope $(\mathrm{A} \beta$ residues $18-23$ ) overlaps with that of crenuzumab [32]. Crenezumab is a passive $A D$ immunotherapy targeting multiple forms of $A \beta$ [32], which is currently in phase three clinical trials [26, 29]. We also examined IVIg, a polyclonal source of human immunoglobulins not specifically targeting $\mathrm{A} \beta$, but also previously trialed as an $\mathrm{AD}$ therapeutic [33]. Accuracy of HPLC-MS/MS of A $\beta 42$ and A $\beta 40$ was not affected by the presence of either exogenous antibody source. While we only tested one anti-A $\beta$-antibody, it is anticipated that the method is not generally susceptible to anti-analyte antibody interference based on the methodology (e.g., strong denaturation step, fractionation, and direct detection of the analytes of interest). Thus, the HPLC-MS/MS method can be used not only in routine care, but also in clinical trials involving anti-A $\beta$-therapeutics.

\section{Conclusions}

Evaluation of pre-analytical and analytical challenges in the context of HPLC-MS/MS improved method accuracy, sensitivity, robustness, and stability for both $A \beta 42$ and
A 340 peptides, and enabled development of CSF color/ clarity acceptance/rejection criteria. To optimize analyte recovery in the analytical phase, we recommend the use of a polypropylene plate with either a low protein binding designation or pre-treated with a BSA solution. For the SPE step, significant gains were made by altering the composition and volume of the wash and elution steps. For the surrogate matrix for the external calibrators, supplementation with BSA in the range of $1-2 \mathrm{~g} / \mathrm{L}$ was found to be optimal, where we ultimately used $1 \mathrm{~g} / \mathrm{L}$ based on physiological CSF total protein concentrations observed in the intended population.

Research funding: LF is supported by a fellowship from the Canadian Institutes for Health Research (No. 201911FBD434952-295330). MLD acknowledges funding from the Michael Smith Foundation for Health Research (MSFHR, No. 16353) and Brain Canada through the Canada Brain Research Fund with the financial support of Health Canada, MSFHR, University of British Columbia's Faculty of Medicine and the Djavad Mowafaghian Centre for Brain Health, Women's Brain Health Initiative, and the St Paul's Foundation.

Author contributions: All authors contributed to the drafting and revision of this work and approved the final version of the manuscript.

Competing interests: The authors declare that they have no known financial or personal competing interests that would influence the work outlined in this paper.

Informed consent: Not applicable.

Ethical approval: Research involving human subjects complied with all relevant national regulations, institutional policies and is in accordance with the tenets of the Helsinki Declaration (as revised in 2013) and was carried out with Research Ethics Board approval from Providence Health Care Research Institute (H18-03050).

\section{References}

1. Hampel H, Frank R, Broich K, Teipel SJ, Katz RG, Hardy J, et al. Biomarkers for Alzheimer's disease: academic, industry and regulatory perspectives. Nat Rev Drug Discov 2010;9:560-74.

2. Jack CR, Jr., Knopman DS, Jagust WJ, Shaw LM, Aisen PS, Weiner MW, et al. Hypothetical model of dynamic biomarkers of the Alzheimer's pathological cascade. Lancet Neurol 2010;9:119-28.

3. Dubois B, Feldman HH, Jacova C, Hampel H, Molinuevo JL, Blennow $\mathrm{K}$, et al. Advancing research diagnostic criteria for Alzheimer's disease: the IWG-2 criteria. Lancet Neurol 2014;13:614-29.

4. Jack CRJ, Bennett DA, Blennow K, Carrillo MC, Dunn B, Haeberlein $S B$, et al. NIA-AA research framework: toward a biological definition of Alzheimer's disease. Alzheimers Dement 2018;14:535-62. 
5. Jack CRJ, Bennett DA, Blennow K, Carrillo MC, Feldman HH, Frisoni $\mathrm{GB}$, et al. $\mathrm{A} / \mathrm{T} / \mathrm{N}$ : an unbiased descriptive classification scheme for Alzheimer's disease biomarkers. Neurology 2016;87:539-47.

6. Mo J. Cerebrospinal fluid amyloid- $\beta$ 1-42 levels in the differential diagnosis of Alzheimer's disease - systematic review and metaanalysis. Value Health 2014;17:391.

7. Slemmon JR, Shapiro A, Mercken M, Streffer J, Romano G, Andreasen $\mathrm{N}$, et al. Impact of cerebrospinal fluid matrix on the detection of Alzheimer's disease with $A \beta 42$ and influence of disease on the total-A $\beta 42 / A \beta 40$ ratio. J Neurochem 2015;135: 1049-58.

8. Selkoe DJ. Alzheimer's disease: genes, proteins, and therapy. Physiol Rev 2001;81:741-66.

9. Toombs J, Foiani MS, Wellington H, Paterson RW, Arber C, Heslegrave A, et al. Amyloid- $\beta$ peptides are differentially vulnerable to preanalytical surface exposure, an effect incompletely mitigated by the use of ratios. Alzheimer's Dementia (Amst) 2018;10:311-21.

10. Stine WB, Jungbauer $L, Y u C$, LaDu MJ. Preparing synthetic $A \beta$ in different aggregation states. Methods Mol Biol 2011;670:13-32.

11. Janelidze S, Stomrud E, Brix B, Hansson O. Towards a unified protocol for handling of CSF before $\beta$-amyloid measurements. Alzheimer's Res Ther 2019;11:63.

12. Rózga M, Bittner T, Batrla R, Karl J. Preanalytical sample handling recommendations for Alzheimer's disease plasma biomarkers. Alzheimer's Dementia (Amst) 2019;11:291-300.

13. Vanderstichele HM, Teunissen CE, Vanmechelen E. Critical steps to be taken into consideration before quantification of $\beta$-amyloid and tau isoforms in blood can be implemented in a clinical environment. Neurol Ther 2019;8:129-45.

14. Naithani $M$, Singh P. Teitz textbook of clinical chemistry \& molecular diagnostics. Med J Armed Forces India 2006;62:204.

15. Bittner T, Zetterberg H, Teunissen CE, Ostlund RE Jr., Militello M, Andreasson U, et al. Technical performance of a novel, fully automated electrochemiluminescence immunoassay for the quantitation of $\beta$-amyloid (1-42) in human cerebrospinal fluid. Alzheimer's Dementia 2016;12:517-26.

16. Bjerke M, Portelius E, Minthon L, Wallin A, Anckarsater H, Anckarsater $\mathrm{R}$, et al. Confounding factors influencing amyloid- $\beta$ concentration in cerebrospinal fluid. Int J Alzheimer's Dis 2010; 2010:986310.

17. Hansson O, Mikulskis A, Fagan AM, Teunissen C, Zetterberg H, Vanderstichele $\mathrm{H}$, et al. The impact of preanalytical variables on measuring cerebrospinal fluid biomarkers for Alzheimer's disease diagnosis: a review. Alzheimers Dement 2018;14: 1313-33.

18. Leitão MJ, Baldeiras I, Herukka S-K, Pikkarainen M, Leinonen V, Simonsen AH, et al. Chasing the effects of pre-analytical confounders - a multicenter study on CSF-AD biomarkers. Front Neurol 2015;6. https://doi.org/10.3389/fneur.2015.00153.

19. Korecka M, Waligorska T, Figurski M, Toledo JB, Arnold SE, Grossman M, et al. Qualification of a surrogate matrix-based absolute quantification method for amyloid- $\beta_{42}$ in human cerebrospinal fluid using 2D UPLC-tandem mass spectrometry. J Alzheimer's Dis 2014;41:441-51.

20. Lame ME, Chambers EE, Blatnik M. Quantitation of amyloid- $\beta$ peptides $A \beta 1-38, A \beta 1-40$, and $A \beta 1-42$ in human cerebrospinal fluid by ultra-performance liquid chromatography-tandem mass spectrometry. Anal Biochem 2011;419:133-9.

21. Leinenbach A, Pannee J, Dulffer T, Huber A, Bittner T, Andreasson $\mathrm{U}$, et al. Mass spectrometry-based candidate reference measurement procedure for quantification of amyloid- $\beta$ in cerebrospinal fluid. Clin Chem 2014;60:987-94.

22. CLSI. Interference testing in clinical chemistry; approved guideline. CLSI document EP-07A, Vol. 3. Wayne, PA: Clinical and Laboratory Standards Institute; 2005.

23. Amatuzio DS, Weber LJ, Nesbitt S. Bilirubin and protein in the cerebrospinal fluid of jaundiced patients with severe liver disease with and without hepatic coma. J Lab Clin Med 1953;41:615-8.

24. Burke BJ, McKee JI, Hargreaves T. Hyperlipidaemia diagnosed at lumbar puncture. Postgrad Med J 1981;57:113-4.

25. Seehusen DA, Reeves MM, Fomin DA. Cerebrospinal fluid analysis. Am Fam Physician 2003;68:1103-8.

26. Cummings JL, Cohen S, van Dyck CH, Brody M, Curtis C, Cho W, et al. A phase 2 randomized trial of crenezumab in mild to moderate Alzheimer's disease. Neurology 2018;90:1889-97.

27. Vanderstichele H, Stoops E, Vanmechelen E, Jeromin A. Potential sources of interference on amyloid- $\beta$ immunoassays in biological samples. Alzheimer's Res Ther 2012;4:39.

28. DeMarco ML, Nguyen Q, Fok A, Hsiung GYR, van der Gugten JG. An automated clinical mass spectrometric method for identification and quantification of variant and wild-type amyloid- $\beta$ 1-40 and 1-42 peptides in CSF. Alzheimers Dement 2020;12:e12036.

29. Salloway S, Honigberg LA, Cho W, Ward M, Friesenhahn M, Brunstein F, et al. Amyloid positron emission tomography and cerebrospinal fluid results from a crenezumab anti-amyloid- $\beta$ antibody double-blind, placebo-controlled, randomized phase II study in mild-to-moderate alzheimer's disease (BLAZE). Alzheimer's Res Ther 2018;10:96.

30. Hooshfar S, Basiri B, Bartlett MG. Development of a surrogate matrix for cerebral spinal fluid for liquid chromatography/mass spectrometry based analytical methods. RCM (Rapid Commun Mass Spectrom) 2016;30:854-8.

31. McCudden CR, Brooks J, Figurado P, Bourque PR. Cerebrospinal fluid total protein reference intervals derived from 20 years of patient data. Clin Chem 2017;63:1856-65.

32. Adolfsson O, Pihlgren M, Toni N, Varisco Y, Buccarello AL, Antoniello $K$, et al. An effector-reduced anti- $\beta$-amyloid $(A \beta)$ antibody with unique $A \beta$ binding properties promotes neuroprotection and glial engulfment of $A \beta$. J Neurosci: Off J Soc Neurosci 2012;32:9677-89.

33. Relkin NR, Thomas RG, Rissman RA, Brewer JB, Rafii MS, van Dyck $\mathrm{CH}$, et al. A phase 3 trial of intravenous immunoglobulin for Alzheimer's disease. Neurology 2017;88:1768-75. 